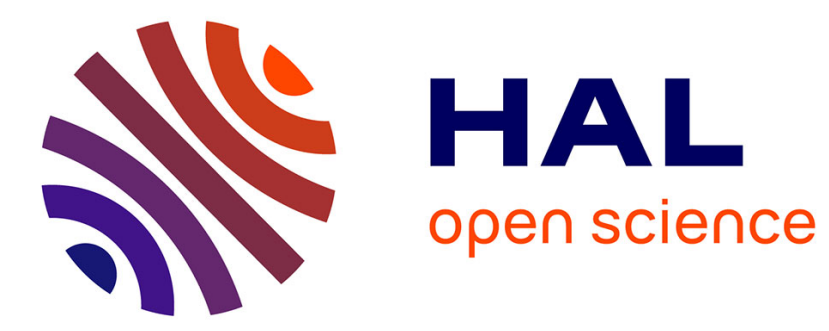

\title{
Low-temperature strength tests and SEM imaging of hydroxide catalysis bonds in silicon
}

N L Beveridge, a A van Veggel, M Hendry, P Murray, R A Montgomery, E Jesse, J Scott, R B Bezensek, L Cunningham, J Hough, et al.

\section{To cite this version:}

N L Beveridge, a A van Veggel, M Hendry, P Murray, R A Montgomery, et al.. Low-temperature strength tests and SEM imaging of hydroxide catalysis bonds in silicon. Classical and Quantum Gravity, 2011, 28 (8), pp.85014. 10.1088/0264-9381/28/8/085014 . hal-00690083

\section{HAL Id: hal-00690083 https://hal.science/hal-00690083}

Submitted on 21 Apr 2012

HAL is a multi-disciplinary open access archive for the deposit and dissemination of scientific research documents, whether they are published or not. The documents may come from teaching and research institutions in France or abroad, or from public or private research centers.
L'archive ouverte pluridisciplinaire HAL, est destinée au dépôt et à la diffusion de documents scientifiques de niveau recherche, publiés ou non, émanant des établissements d'enseignement et de recherche français ou étrangers, des laboratoires publics ou privés. 


\title{
Low temperature strength tests and SEM imaging of hydroxide-catalysis bonds between silicon
}

\author{
N L Beveridge ${ }^{1}$, A A van Veggel ${ }^{1}$, M Hendry $^{1}$, P Murray $^{1}, \mathbf{R}$ A \\ Montgomery $^{1}$, E Jesse ${ }^{2}$ J Scott $^{1}$, R B Bezensek ${ }^{3}$, L Cunningham ${ }^{1}$, J \\ Hough $^{1}$, R Nawrodt ${ }^{1,4}$ S Reid ${ }^{1}$ S Rowan ${ }^{1}$, \\ ${ }^{1}$ SUPA Institute for Gravitational Research, School of Physics and Astronomy, University of \\ Glasgow, Glasgow G12 8QQ, UK \\ 2 Embry-Riddle Aeronautical University - Prescott, 3700 Willow Creek Road, Prescott, AZ \\ 86301-3720, USA \\ ${ }^{3}$ Hunting Energy Services UK, Aberdeen, AB12 4YB \\ ${ }^{4}$ Institute of Solid State Physics, Friedrich-Schiller-Universität Jena, 07743 Jena, Germany \\ E-mail: n.beveridge@physics.gla.ac.uk
}

\begin{abstract}
Silicon is under consideration as a substrate material for the test masses and suspension elements of gravitational wave detectors of improved sensitivity. Hydroxide catalysis bonding is a candidate technique for jointing silicon elements with the potential for both high strength and low mechanical loss. A future detector with quasi-monolithic silicon final stages may operate at cryogenic temperatures. Here we present the first studies of the strength of siliconsilicon bonds at $77 \mathrm{~K}$ (liquid nitrogen temperature) and show characteristic strengths of $\sim 44 \mathrm{MPa}$ are achieved. When comparing cryogenic to room temperature results no significant difference is apparent in the strength. We also show that a minimum thickness of oxide layer of $50 \mathrm{~nm}$ is desirable to achieve reliably strong bonds. Bonds averaging $47 \mathrm{~nm}$ in thickness are achieved for oxide thicknesses greater than $50 \mathrm{~nm}$.
\end{abstract}

\section{Introduction}

Hydroxide catalysis bonding is a technique that was developed and used successfully for the telescope construction in the Gravity Probe B space mission [?]. This technology has successfully been adapted for use in the construction of the mirror suspensions of laser interferometric gravitational observatories. A network of six detectors has been constructed to search for gravitational waves from astrophysical events that involve the asymmetric acceleration of significant mass. There are three detectors (two $4 \mathrm{~km}$ detectors and one $2 \mathrm{~km}$ detector) forming the US LIGO system [?, ?], a French-Italian detector, Virgo [?], the German-UK GEO 600 detector [?] and the TAMA300 instrument [?] in Japan. These detectors aim to measure the change in separation of mirrors suspended as pendulums caused by astrophysical gravitational radiation.

The technique of hydroxide catalysis bonding was applied in the GEO 600 detector in order to create a quasi-monolithic suspension of the fused silica mirrors. Fused silica fibres were welded to fused silica interface pieces, called 'ears' which provide suitable welding points onto the sides of the mirror, [?] bonded onto the sides of the mirror using the hydroxide catalysis bonding technique [?]. The technique will be used in a similar way in upgrades to the suspensions of the LIGO [?] and Virgo [?] detectors to form 'Advanced' detector systems which aim to be operational around 2014. These 
advanced instruments should have a factor of 10 to 15 times better sensitivity than first generation detectors. The reason for using 'monolithic' fused silica mirror suspensions is to reduce as much as possible the thermal noise in the operating frequency band of the detectors between $\sim 10 \mathrm{~Hz}$ and a few $\mathrm{kHz}$, which can be achieved by reducing the number of contact points of the suspension fibres to the test masses or making any interfaces low loss. Fused silica is a material that shows suitably low thermal noise at room temperature [?, ?, ?]. The hydroxide catalysis bonding technique adds very little to the noise, because of a combination of its level of mechanical loss [?, ?], small bond thickness and high mechanical strength [?]. Additionally it is vacuum compatible and allows for precision alignment of pieces.

Plans are also developing for the design of potential 'third generation' detectors (such as the Einstein Telescope (http://www.et-gw.eu)) [?, ?]. These detectors may well operate at cryogenic temperatures to further reduce thermal noise. Silicon is a prime candidate material for use in the test masses and their suspensions because of its desirable thermo-mechanical properties in the cryogenic regime [?, ?, ?, ?]. With some adaptation, hydroxide catalysis bonding may also be a viable technique for use in third generation detectors; however, to evaluate its suitability it is essential to quantify both the strength of silicon-silicon bonds at cryogenic temperatures and the thickness of such bonds as the bond thickness has a direct effect on the contributions of the bond to the overall thermal noise of a bonded suspension.

We have previously investigated the strength of silicon-silicon bonds at room temperature under shear [?] with some investigation into the effect of the oxide layer. Other authors [?] have studied the effect of the effective flatness of silicon and sapphire bonds on bending strengths of hydroxide catalysis bonds at room temperature. In this paper investigations of the strength of silicon-silicon hydroxide catalysis bonds are presented applying for the first time the same strength testing technique as was used in [?] both at room temperature and at $\sim 77 \mathrm{~K}$, allowing studies of the strength of the bonds to be carried out as a function of the thickness of the thermal $\mathrm{SiO}_{2}$ layer grown on the surface to be bonded.

\section{Hydroxide Catalysis Bonding}

The hydroxide catalysis bonding technique allows bonding between a variety of materials if a silicatelike network can be formed at the material surfaces, which is the case for most oxide materials, e.g. silica, Zerodur, fused silica, ULE glass or granite [?]. The two materials are bonded using an alkaline bonding solution; e.g. sodium hydroxide $(\mathrm{NaOH})$, potassium hydroxide $(\mathrm{KOH})$ or sodium silicate $\left(\mathrm{Na}_{2} \mathrm{SiO}_{3}\right)$ dissolved in water.

For application to the suspensions of the gravitational wave detectors the bonding surfaces ideally have global surface figures that match to within $60 \mathrm{~nm}$ peak-to-valley to create very thin bonds with a hydroxide solution; the final thickness of the bond is important in minimizing the mechanical loss and associated thermal noise. Both bonding surfaces are cleaned in a clean room environment to remove any chemical or particulate contaminants. The bonding solution is filtered with a $0.2 \mu \mathrm{m}$ pore size filter and a volume of typically a few tenths of $\mu \mathrm{l} / \mathrm{cm}^{2}$ is deposited on one bonding surface (facing upwards). The other substrate is then placed gently on top of the first substrate and can be compressed slightly to aid successful bonding. When the bonding solution comes into contact with the bonding surfaces the hydroxide catalysis commences. This consists of three steps; hydration and etching, polymerization, and dehydration [?]. These steps have been described in detail in previous papers [?, ?].

The time taken for a bond to form can be controlled by altering temperature and/or $\mathrm{pH}$ of 
the bonding solution used [?,?] . The bond thickness between fused silica surfaces is found to be approximately $40 \mathrm{~nm}-100 \mathrm{~nm}[?, ?]$.

To make bonding of silicon components possible, the bonding surfaces must ideally have a thin coating of $\mathrm{SiO}_{2}$, with which the hydroxide can react to form the bond. This layer can be formed, for example, through thermal oxidation by placing the cleaned pieces in a quartz tube furnace at typically $800-1200^{\circ} \mathrm{C}$ in a dry air or a wet environment using air, nitrogen or oxygen as a carrier gas [?]. Previous studies of bonding silicon and silicon carbide [?] seemed to suggest that the nature of the silicon oxide layer plays a role in the success of the bonding process. In [?] it was postulated that if hydrogen formed during the bonding process due to direct contact between silicon and hydroxide this might have an effect on strength.

In practical configurations in the suspensions of gravitational wave detectors the forces applied to bonds are not purely shear; 'peel' stresses are also present, thus the strength of bonds under different types of loading conditions are of interest.

\section{Strength Testing}

For the strength tests rectangular silicon blocks $(20 \mathrm{~mm} \times 10 \mathrm{~mm} \times 5 \mathrm{~mm})$ from two different silicon ingots were used. Both ingots were manufactured by Prolog; ingot one had a $<111>$ crystal orientation, ingot two had a $\langle 100>$ crystal orientation, as shown in Figure 1 . Both were boron doped P-type silicon ingots with an unknown doping level. The bonding surface was one of the $5 \mathrm{~mm} \times 10 \mathrm{~mm}$ surfaces, so that after bonding a sample was created with dimensions $40 \mathrm{~mm} \times 10 \mathrm{~mm} \times 5 \mathrm{~mm}$.

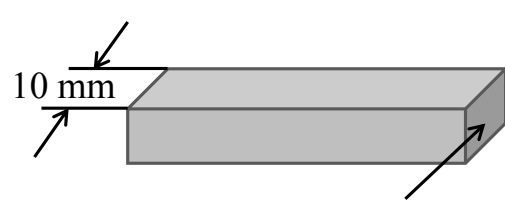

(100) plane

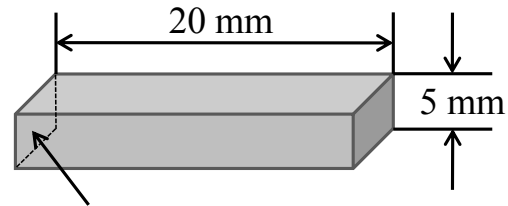

(111) plane
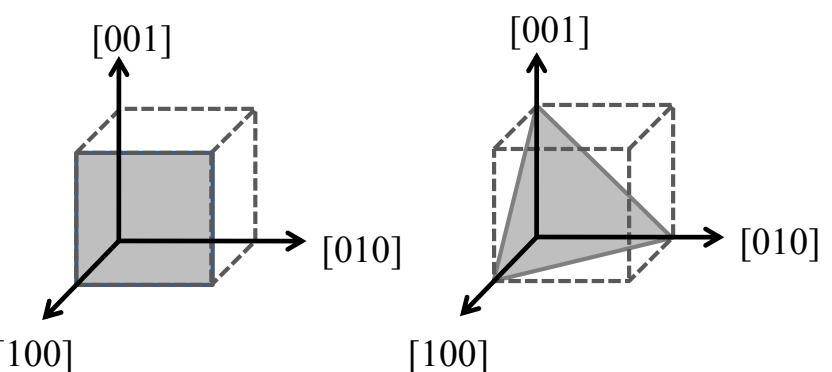

[100]

$[100]$

Figure 1. Diagram showing a 'mixed' ingot pairing. The bonding surface on each block is $5 \mathrm{~mm}$ $\mathrm{x} 10 \mathrm{~mm}$, and for a mixed ingot pairing, a $<111>$ block is bonded to a block of orientation $<100>$

The bonding surfaces of all samples were polished to a nominal flatness of $\lambda / 10$ where 
$\lambda=633 \mathrm{~nm}$. The flatness of each side was measured before oxidation using a Zygo GPI XP/D $\ddagger$ interferometer at $633 \mathrm{~nm}$. All samples were marked with identification numbers, to allow the bonds to be characterized and tracked throughout the experimental process.

\subsection{Sample Preparation}

In order to remove any organic residue on the bonding surfaces prior to oxidation an acid clean, using a 1:7 volumetric ratio of hydrogen peroxide and sulphuric acid, was performed on all samples.

The samples were placed in a tube furnace at $\sim 1000^{\circ} \mathrm{C}$ for periods of time between 5 and 55 minutes to grow thermal $\mathrm{SiO}_{2}$ layers of various thicknesses. A wet nitrogen method was used; nitrogen gas saturated with water vapor was passed through the furnace for the duration of the oxidation.

During the process of oxidising some of the blocks, it was discovered that the oxide layers grown were not as thick as originally intended. As a result, it was necessary to re-oxidise some of the samples. The oxidised blocks were put back into the tube furnace (in the same environment as before) to 'top-up' the oxide layer. The resulting oxide layer was measured and assumed to be a single oxide layer.

The bonding surfaces were made hydrophilic prior to bonding through a regime of polishing with cerium oxide in de-ionised water followed by cleaning with sodium bicarbonate. Immediately before the bonding solution was deposited, the surface was wiped with methanol as a final cleaning step.

The bonding solution was pipetted onto the surfaces using $0.4 \mu \mathrm{l}$ of solution per $\mathrm{cm}^{2}(0.2 \mu \mathrm{l}$ for the strength test samples). The bonding solution was produced by diluting a commercially available sodium silicate solution $\left(14 \% \mathrm{NaOH}\right.$ and $\left.27 \% \mathrm{SiO}_{2}\right)$ with de-ionized water at a volumetric ratio of 1:6, a solution used previously [?].

All samples were left to cure at room temperature for a minimum period of 4 weeks and were categorized by the minimum oxide layer, $t_{\min }$, of the pair of surfaces to be bonded, the difference between the thickness of each of the two oxide layers, $t_{\text {diff }}$ and the ingot pairing.

\subsection{Strength Testing Procedure}

The bond strength was determined using a four point bend test as shown in Figure 2 and is in line with ASTM standard C 1161-2C [?]. The samples were placed on the setup and a force, $F$, was applied through a loading arm and measured until the sample broke. If the supports underneath the sample are placed at a distance $L$ the force is applied equally through two points on top of the sample at a distance $L / 2$ apart, with both distances centered at the bond.

The samples were tested on Zwick-Roell static $2 \mathrm{kN}$ and $200 \mathrm{kN}$ machines, and the strength was calculated using (1) below where $F$ is the force, $L$ is the distance between the bottom supports, $b$ is the width of the sample and $d$ is the thickness of the sample.

$$
\sigma_{\max }=\frac{3 F L}{4 b d^{2}}
$$

Measurements were performed at room temperature and at $\sim 77 \mathrm{~K}$. The latter cryogenic measurements were performed by submerging the samples in liquid nitrogen and waiting for thermal equilibrium by observing the settling of the nitrogen after boiling before conducting the strength test.

$\ddagger$ http://www.zygo.com/?/met/interferometers/gpi/ 


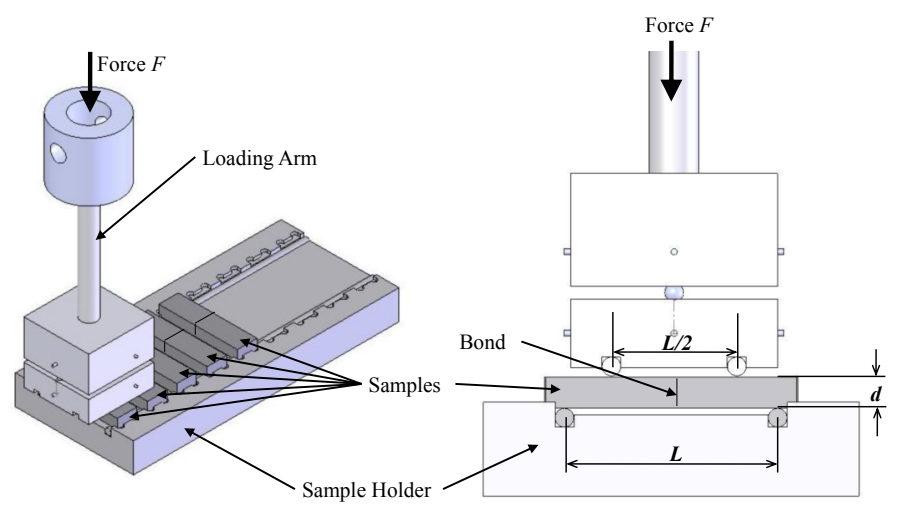

Figure 2. 3D and detail CAD images of the bending strength set-up. The sample holder slides underneath the loading arm to reduce time needed between testing samples.

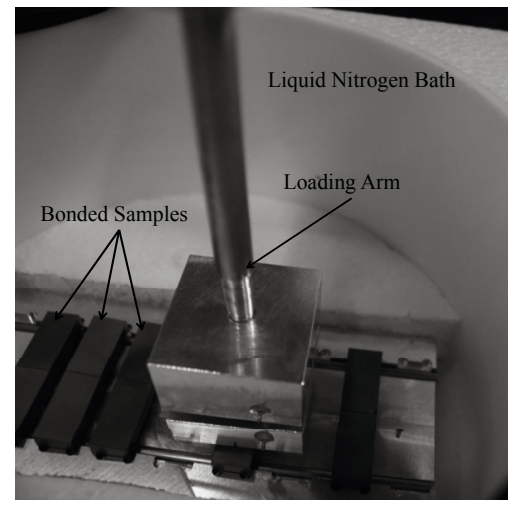

Figure 3. Image showing the test set up; to cool the samples down to $\sim 77 \mathrm{~K}$, the bath was filled with liquid nitrogen.

109 samples were strength tested; 49 at room temperature and 86 at $\sim 77 \mathrm{~K}$. A photograph of the strength testing is shown in Figure 3.

\subsection{Results}

Figure 4 shows how the strength of the samples varied with the minimum oxide layer thickness $\left(t_{\min }\right)$ found on the surface of each sample and three main features can be identified. Firstly, when looking only at the cryogenic temperature results, there is a clear trend of decreasing strength once the minimum oxide layer is less than $50 \mathrm{~nm}$. Secondly, when comparing cryogenic to room temperature results at comparable oxide layer thicknesses there appears to be no significant difference apparent in strengths indicating that the strength of the bonds is not negatively influenced by thermal loading due to expansion differences between the bond material (silicon dioxide and siloxane chains) and the bulk material (silicon). Lastly, there appears to be a grouping of samples that lie in $75 \mathrm{~nm}<t_{\min }<260 \mathrm{~nm}$ that have somewhat lower strength (less than $30 \mathrm{MPa}$ ) than the remainder 


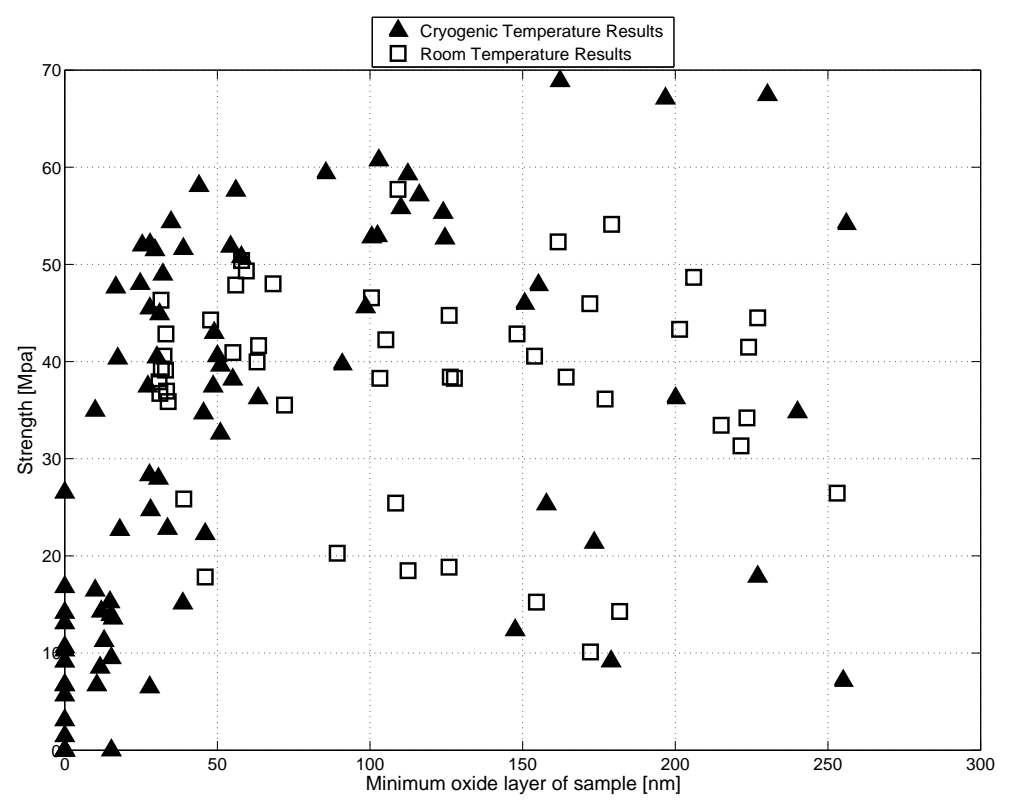

Figure 4. Graphical representation of all results. The bond strength at cryogenic temperature shows a similar average strength as at room temperature, but a wider range of strengths is apparent. The absence of correlation between oxide layer thickness and bond strength at thicknesses above $\sim 50 \mathrm{~nm}$ shows an opportunity to minimize potential thermal noise by reducing the oxide layer thickness as much as possible without compromising bond strength.

of the samples.

Due to the brittleness of the samples, a statistical variation could be expected to be seen in their strengths. This occurs because a fracture may originate at flaws in the material itself and therefore the strength will depend upon the probability of there being a flaw that is large enough to cause a fracture normal to the direction of the maximum tensile stress.

The variations in strength of such materials are generally modeled by a Weibull distribution [?] rather than, for example, a Gaussian. The Weibull distribution is commonly used in modeling extreme or critical phenomena or in survival and reliability analysis where one wishes to estimate the 'time to failure' of an industrial or mechanical process. In particular the Weibull distribution can be strongly skewed and is thus appropriate for modeling statistical variations which are not symmetric with respect to some given characteristic behaviour - such as one may encounter when considering the distribution of breaking strengths.

The two-parameter Weibull distribution has a probability density function (pdf) given by (2):

$$
P(F)=\frac{m}{F_{0}}\left(\frac{F}{F_{0}}\right)^{m-1} \exp \left[-\left(\frac{F}{F_{0}}\right)^{m}\right]
$$

where the Weibull modulus, $m$, provides a measure of the variability of the material properties - with increasing Weibull modulus indicating a smaller range of variability. The parameter $F_{0}$ represents a characteristic strength for the distribution. The combination of these two parameters 
permits comparison between data sets acquired from similar experiments, in order to explore and identify factors that might influence the strength of the data sets.

In previous literature (see [?]) estimation of $m$ and $F_{0}$ has generally been carried out in terms of the cumulative distribution that corresponds to the pdf of (2). The measured strengths and estimated cumulative probabilities for the data set are transformed into new variables related to $m$ and $F_{0}$ via a simple straight line dependence. Fits to these parameters are then obtained via, for example, linear regression. However this approach generally assumes that the scatter about the regression line is Gaussian - an assumption that may be questionable given the highly skewed nature of the original Weibull distribution.

In this paper instead a Bayesian analysis was carried out that simultaneously estimates $m$ and $F_{0}$ directly from the Weibull pdf and requires no such Gaussian assumption. Moreover we use a Markov Chain Monte Carlo method [?] to obtain confidence regions for our fitted Weibull parameters. Figure 5 shows $68 \%$ confidence regions for the joint parameter fits to the full cryogenic data set and room temperature data set. Note that these regions are not elliptical in shape, as the Gaussian assumption would require.

Figure 6 shows estimates of the $m$ parameter (top figure) and $F_{0}$ parameter (bottom figure) for all data sets, determined by marginalizing the joint posterior distribution of $m$ and $F_{0}$. Here the markers indicate the mode of the marginalized posterior and the error bars indicate the central $68 \%$ of the distribution. It can be seen that the values for the Weibull modulus and characteristic strength vary when different factors are taken into account. The low value of $m$ for the full data sets indicates that there may be several factors affecting the strength of the samples. Some possible factors were identified during the experiment and filters were applied in order to identify which of these factors influence the characteristic strength and spread of results. Initially, a minimum oxide layer thickness filter was applied. The value of $50 \mathrm{~nm}$ was selected after inspection of Figure 4 as discussed earlier, in which a clustering of low strength samples for oxide thicknesses below $50 \mathrm{~nm}$ can be seen.

Table 1. Data set sizes used in Figure 6

\begin{tabular}{lllc}
\hline Temperature & Filter 01 & Filter 02 & Data Set Size \\
\hline $77 \mathrm{~K}$ & & & 86 \\
$293 \mathrm{~K}$ & & & 49 \\
\hline $77 \mathrm{~K}$ & $t_{\min }>50 \mathrm{~nm}$ & & 33 \\
$293 \mathrm{~K}$ & $t_{\min }>50 \mathrm{~nm}$ & & 37 \\
\hline $77 \mathrm{~K}$ & $t_{\min }>50 \mathrm{~nm}$ & $t_{\text {diff }}>20 \mathrm{~nm}$ & 7 \\
$293 \mathrm{~K}$ & $t_{\min }>50 \mathrm{~nm}$ & $t_{\text {diff }}>20 \mathrm{~nm}$ & 17 \\
\hline $77 \mathrm{~K}$ & $t_{\min }>50 \mathrm{~nm}$ & Diagonal Break & 29 \\
$293 \mathrm{~K}$ & $t_{\min }>50 \mathrm{~nm}$ & Diagonal Break & 33 \\
\hline $77 \mathrm{~K}$ & $t_{\min }>50 \mathrm{~nm}$ & Bond Break & 4 \\
$293 \mathrm{~K}$ & $t_{\min }>50 \mathrm{~nm}$ & Bond Break & 5 \\
\hline $77 \mathrm{~K}$ & $t_{\min }>50 \mathrm{~nm}$ & Re-oxidised & 16 \\
$293 \mathrm{~K}$ & $t_{\min }>50 \mathrm{~nm}$ & Re-oxidised & 14 \\
\hline $77 \mathrm{~K}$ & $t_{\min }>50 \mathrm{~nm}$ & Not Re-oxidised & 17 \\
$293 \mathrm{~K}$ & $t_{\min }>50 \mathrm{~nm}$ & Not Re-oxidised & 24 \\
\hline $77 \mathrm{~K}$ & $t_{\min }>50 \mathrm{~nm}$ & Same Ingot & 25 \\
$293 \mathrm{~K}$ & $t_{\min }>50 \mathrm{~nm}$ & Same Ingot & 20 \\
\hline $77 \mathrm{~K}$ & $t_{\min }>50 \mathrm{~nm}$ & Mixed Ingot & 8 \\
$293 \mathrm{~K}$ & $t_{\min }>50 \mathrm{~nm}$ & Mixed Ingot & 18 \\
\hline
\end{tabular}




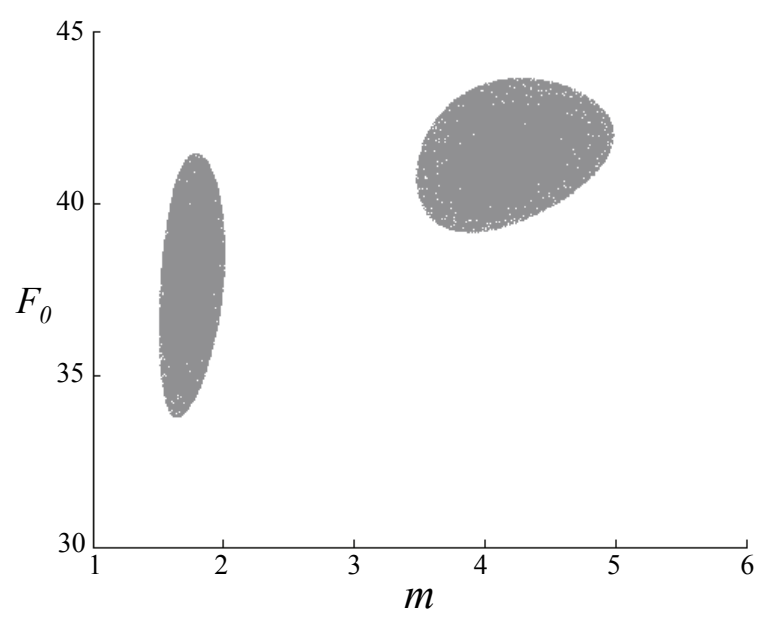

Figure 5. Joint $68 \%$ confidence regions for the Weibull parameters $m$ and $F_{0}$, obtained from a Bayesian analysis of all cryogenic (left) and room temperature (right) strength results.

There appears to be no correlation between the minimum oxide layer thickness on the pair of bonding surfaces for each sample and the strength of the sample when the minimum oxide layer thickness on the bonding surfaces is between $50 \mathrm{~nm}$ and $250 \mathrm{~nm}$. This is of importance because the goal is to minimize the thickness of the overall oxide and bond layer as much as possible for application in third generation gravitational wave detectors, due to the direct relationship between the thickness of the oxide layer and bond and the thermal noise caused by the bond [?].

A significant limitation with this analysis is the small number of data points after certain filters are applied. However, a clear effect is seen in Figure 6 when removing samples with a minimum oxide layer thickness of less than $50 \mathrm{~nm}$ at cryogenic temperatures: both the characteristic strength and Weibull modulus increase. This is not seen at room temperature, possibly because only a very small portion of the room temperature samples had oxide layers below $50 \mathrm{~nm}$. The complete sample set size having oxide thicknesses lower than $50 \mathrm{~nm}$ was too small to allow statistically significant results to be obtained at both room and low $\mathrm{T}$, thus measurements were predominantly made at cryogenic temperature for this group of samples.

The samples broke in two different ways; in a 'bond' break, where the fracture was along the bond of the sample, or in a 'diagonal' break, where the fracture went through the bulk material. It was observed that the majority of the samples with a minimum oxide layer of less than $50 \mathrm{~nm}$ had a bond break in comparison to the samples with thicker oxide layers mostly having a diagonal break pattern, suggesting that when the bonds were weak the sample would break along the bond. This confirms suspicions that there is a minimum oxide layer needed.

Looking further at Figure 6 it was observed that when the difference between the oxide layers on the bonding surfaces of each sample was greater than $20 \mathrm{~nm}$, although the characteristic strength $\left(F_{0}\right)$ only increases at room temperature (it remains the same at cryogenic temperature) the value of $m$ increases by a significant amount at both temperatures, showing a reduction in the spread of strengths. The low grouping seen in Figure 5 is also eliminated. It is hypothesized that there may 

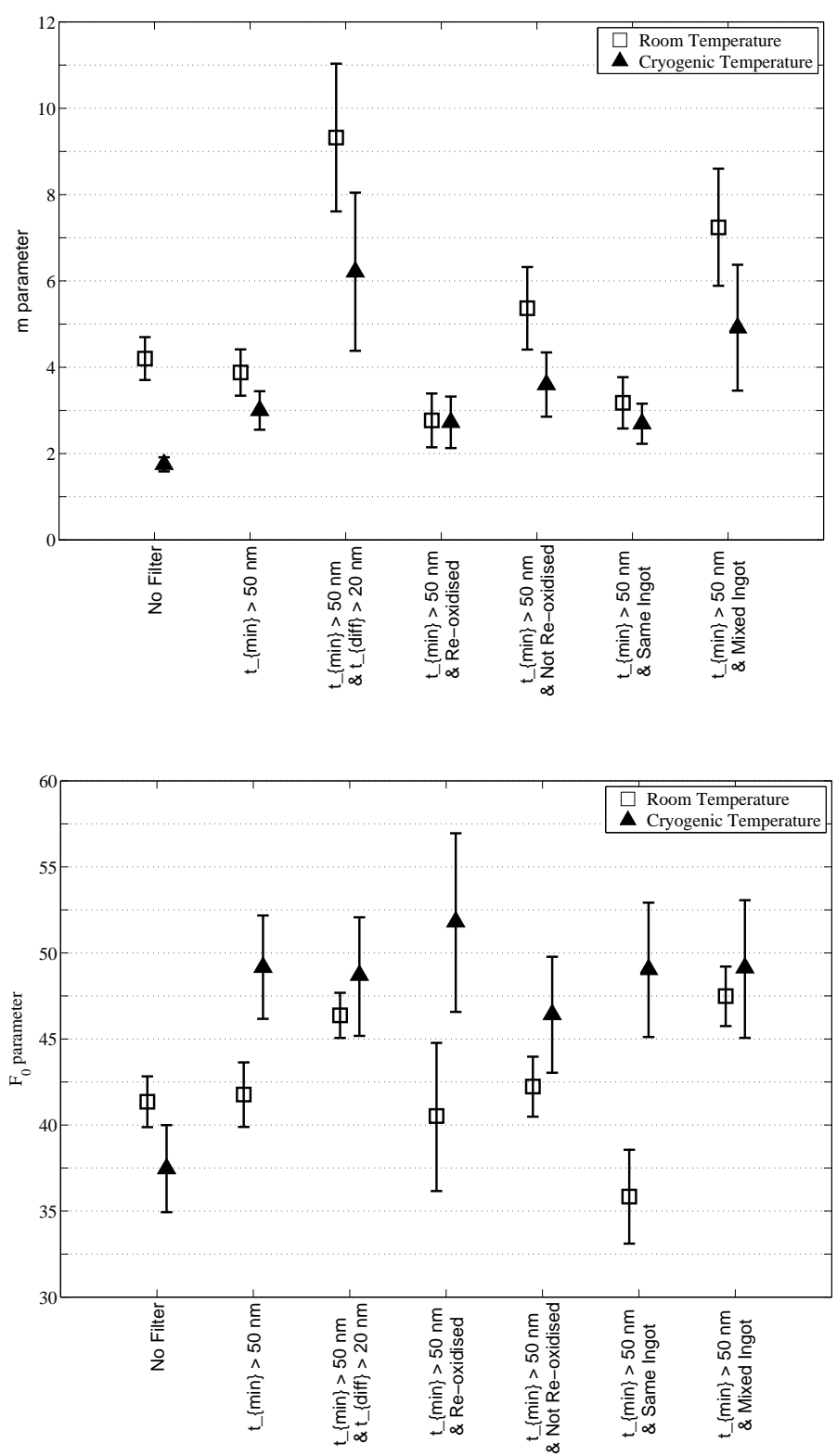

Figure 6. Graphs showing the values for the Weibull modulus (top) characteristic strength (bottom) for each data set, with error bars indicating the $68 \%$ confidence limits. The key describing the filters applied to each data set is shown in Table 1.

be a geometrical effect resulting from the large difference in oxide layer that is influencing these results. Finite Element Analysis may shed light on this. 
The effect of re-oxidation does not consistently change the characteristic strength, however the value of $m$ decreases when the samples were re-oxidised suggesting that the value of the characteristic strength is less reliable when the samples have been re-oxidised. This could be caused by an increased likelihood of contamination of samples before bonding or a weak interface between layers.

There is a marked difference between the room temperature results for characteristic strengths and Weibull modulus of samples from the same ingot in comparison to those from different ingots; both the strength and modulus increase when samples are from mixed ingots. At cryogenic temperature, the strength does not appear to change. However, the Weibull modulus does increase significantly for samples from mixed ingots. A possible explanation is a difference in mechanical behaviour in samples from mixed ingots due to differences in the density of the oxide layer. A slowly grown thermal oxide layer will be denser than one grown rapidly [?]. Silicon of orientation $<111>$ will grow a thermal oxide layer at a faster rate to silicon of orientation $<100>$ and will therefore have a slightly less dense thermal oxide layer. However, the exact mechanism causing the improved value of $m$ and the increase in characteristic strength at room temperature is not yet known.

\section{Bond Thickness}

\subsection{Sample Preparation}

For the measurements of bond thickness 10 silicon discs were used, each of diameter $12.6 \mathrm{~mm}$ and thickness $6.3 \mathrm{~mm}$, from the same ingot as the samples discussed in [?] $(<111>$, purity $>99.999 \%$, resistivity $>1 \mathrm{Ohm} \mathrm{cm})$.

These samples were oxidized using the same wet oxidation technique as for the strength tests, with two samples oxidized for each oxidation time of 10, 20, 30, 40 and 50 minutes, respectively. After oxidation the thicknesses of the oxide layers on each of the silicon samples were measured at several points on the bonding surfaces using a Sentech SE850 Ellipsometer§, and the results averaged. The flatness measurements of the samples were repeated using the Zygo interferometer and the results averaged.

Table 2 shows the measured oxide thicknesses for the discs used in the bond thickness investigation.

Table 2. Oxide thicknesses of silicon discs measured using ellipsometry investigation into bond thickness.

\begin{tabular}{lcc}
\hline Oxidation Time (mins) & $\begin{array}{l}\text { Oxide Thickness of } \\
\text { Side 1 (nm) } \\
\text { (ellipsometry) }\end{array}$ & $\begin{array}{l}\text { Oxide Thickness of } \\
\text { Side 2 (nm) } \\
\text { (ellipsometery) }\end{array}$ \\
\hline 10 & $46 \pm 3$ & $49 \pm 2$ \\
20 & $60 \pm 3$ & $90 \pm 3$ \\
30 & $104 \pm 5$ & $134 \pm 5$ \\
40 & $196 \pm 4$ & $152 \pm 4$ \\
50 & $259 \pm 4$ & $172 \pm 4$ \\
\hline
\end{tabular}

$\S$ http://www.sentech.de/index.php?st_id=15\&itid=219 


\subsection{Scanning Electron Microscope imaging}

From the bonded $12.6 \mathrm{~mm}$ diameter disc samples, two $3 \mathrm{~mm}$ cross sections were sawn in such a way that the bond and oxide layers lay perpendicular to the cut face.

The slices cut from the silicon substrates were then polished with progressively finer polishing grits $(16 \mu \mathrm{m}, 9 \mu \mathrm{m}, 3 \mu \mathrm{m}$, and sub-micron grits) on a Logitech Precision Polishing Machine\|. The surface roughness after polishing is in the order of $\mathrm{Ra} \sim 5 \mathrm{~nm}$ after polishing. This makes it possible to image the bonds using scanning electron microscopy.

The samples were cleaned and studied using a Hitachi TM-1000 Tabletop Electron Microscope and images of the bond and oxide region were captured. This gave a clear indication of the total thickness of the bond and oxide layers. However, when viewed using the maximum magnification of the electron microscope $(10,000)$ it is impossible to readily distinguish between the oxide and bond layers themselves in each of the cross sections. A selection of the cross sections were then imaged using a more powerful FEI Nova 200 Dual beam FIB system Scanning Electron Microscope ${ }^{+}$at the Kelvin Nanocharacterisation Centre at the University of Glasgow.

\subsection{Results of the SEM imaging}

Using the Hitachi tabletop electron microscope it was possible to measure the overall thickness of the bond and oxide layers for all samples mentioned above. With the Nova scanning electron microscope this was possible as well. The results are shown in Table 3. The averages are taken over more than 20 measurements per sample from both the Nova and Hitachi SEMs. The error is the error of the mean of these measurements along the entire length of the sample, and indicates that there was a relatively small amount of variation of thickness along the length of the sample. By subtracting the thicknesses of oxide layers measured prior to bonding (see Table 3), the bond thickness can be determined.

Table 3. Summary of the total bond and oxide thicknesses for the differing oxidation times measured using electron microscopy

\begin{tabular}{ll}
\hline $\begin{array}{l}\text { Oxidation Time } \\
(\text { mins })\end{array}$ & $\begin{array}{l}\text { Total Thickness } \\
( \pm \text { standard error })(\mathrm{nm})\end{array}$ \\
\hline 10 & $131 \pm 9$ \\
20 & $209 \pm 14$ \\
30 & $287 \pm 16$ \\
40 & $388 \pm 18$ \\
50 & $479 \pm 14$ \\
\hline
\end{tabular}

The bond thickness is plotted against oxide layer thickness in Figure 7. There is very little variation in the bond thicknesses with oxidation time or thickness and the average bond thickness was found to be $47 \pm 15 \mathrm{~nm}$. The average peak to valley flatness of the samples after oxidation, measured before bonding was $43 \pm 3 \mathrm{~nm}$.

Figure 8 shows an image of a cross section of a bond between two oxide layers created after a 30 minute oxidation made with the NOVA scanning electron microscope at $75000 \mathrm{x}$ magnification.

\| http://www.logitech.uk.com/PM5.asp

I http://www.hht-eu.com/cms/7929.html

+ http://www.knc.gla.ac.uk/index.php?L1=content\&L2=Equipment\&L3=FIB 


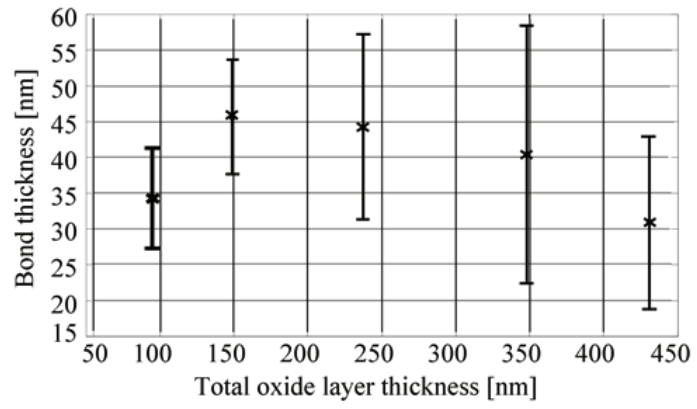

Figure 7. Plot of calculated bond thickness versus total oxide layer thickness for each of the oxidized and bonded silicon samples.

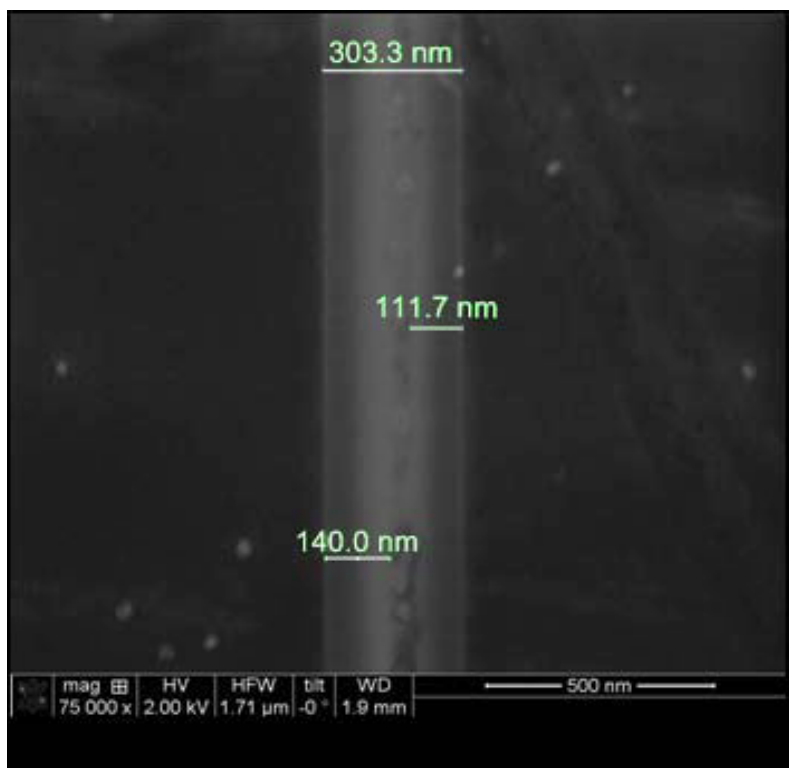

Figure 8. 75,000x magnified Scanning Electron Microscope image of polished cross-section of a 30 minute oxidized hydroxide-catalysis bonded silicon sample.

The image is typical for images made of the other samples. With the NOVA SEM it was also possible to discern the oxide and bond layers. The oxide thickness measurements with the SEM are very comparable (to $\pm 10 \mathrm{~nm}$ ) to the thicknesses measured before bonding using ellipsometry (compared to Table 2). It is interesting to note that the oxide layers have not thinned significantly due to any hydroxide etching. 


\section{Discussion and Summary}

Bend strength tests have been performed on silicon-silicon bonds that incorporated thermal oxide layers of varying thicknesses. The bond strength reduces significantly with oxide layer thicknesses below $50 \mathrm{~nm}$ at cryogenic temperature. Strength test results show a characteristic strength of approximately $49 \mathrm{MPa}$ at $77 \mathrm{~K}$ and $42 \mathrm{MPa}$ at room temperature for samples with a minimum oxide layer of $50 \mathrm{~nm}$.

Van Veggel et al [?] showed bending strengths averaging $23 \mathrm{MPa}$ in silicon carbide-silicon carbide bonds. Those experiments were performed with similar sample geometry and with a very similar 4-point bending set-up. The strengths for silicon-silicon bonds reported by van Veggel et al [?] averaged 3.9 MPa at room temperature. Those strengths are a factor of 10 lower than those reported in this paper. The experiments done by van Veggel et al [?] were however performed under different loading conditions; i.e. they were shear experiments that were thought to be limited by the way the samples were clamped. Performing shear strength experiments that will be less dominated by the sample and loading geometry should shed light on the isotropy of the bond strength under different loading conditions. The results in this paper support the postulation by van Veggel et al [?] that an oxide layer which is too thin can negatively influence the bond strength.

The bending stress in silicon-silicon bonds have previously been measured by Dari et al [?] to be approximately $8.7 \pm 3.7 \mathrm{MPa}$ at room temperature. The results presented here use a different sample geometry and are significantly higher, also demonstrating the need for an oxide layer to be present for a successful bond to form.

In summary, it can be seen that the characteristic strength of all the samples studied at both cryogenic and at room temperature lies above $20 \mathrm{MPa}$, and there is ample opportunity for this to increase along with the Weibull modulus once factors negatively affecting the bond can be identified and removed. It has been shown that repeated oxidation, differences in oxide thickness and ingot pairing can have effects on bond strength that would benefit from further investigation.

The bond thickness measurements show that when the oxide layers on both sample substrates are intact and the oxide layers are at least $50 \mathrm{~nm}$, the bond is uniform in thickness as opposed to wedged (as reported in [?]). This may be because the bonding solution did not come into direct contact with the underlying silicon material, and therefore could not create hydrogen gas bubbles that may induce 'wedging'. The average bond thickness was found to be $47 \mathrm{~nm}$. This value is comparable to the minimal values found in the wedged silicon-silicon samples in [?] and also comparable to values reported by [?] and [?] for silica-silica samples. The fact that the average flatness of the samples after oxidation was $43 \mathrm{~nm}$, a value very close to the average bond thickness, might indicate that there is a direct relation between the flatness of the samples and the obtained bond thickness. This would need to be investigated further.

Further investigation of the factors that affect the bond strength is of interest. The influence of different types of oxidation e.g. dry oxidation and sputtering, and the effect of differences in the oxide layer thickness on bond strength of samples will be considered. The influence of the crystallographic orientation of silicon on the bond strength will also be examined.

\section{Acknowledgments}

We would like to thank John Davidson from the University of Glasgow School of Engineering for his help with the strength measurements, Donald Nicholson from the University of Glasgow School of Engineering for his help with the thermal oxidation of the silicon samples and William Smith for 
his technical support for the SEM imaging.

We would like to thank the Royal Society of Edinburgh, the Science and Technology Facilities Council, the Scottish Funding Council, the EC under contract no. FP7-211743, the Royal Society of London, the Wolfson Foundation and the University of Glasgow for financial support.

Finally, we would like to thank our colleagues in the GEO600 and LSC-Virgo collaborations for their interest in this work.

\section{References}

